\title{
A descentralização como eixo norteador na reorganização e operacionalização dos princípios do Sistema Único de Saúde*
}

\author{
DECENTRALIZATION AS A GUIDING PRINCIPLE FOR THE REORGANIZATIONAND \\ IMPLEMENTATION OF THE UNIFIED HEALTH SYSTEM'S PRINCIPLES
}

\section{LA DESCENTRALIZACIÓN COMO EL EJE NORTEADOR EN LA REORGANIZACIÓN Y OPERACIONALIZACIÓN DE LOS PRINCIPIOS DEL SUS}

\section{Pedro Fredemir Palha1, Tereza Cristina Scatena Villa²}

\section{RESUMO}

A organização do setor saúde no Brasil apresentou mudanças significativas, após a Constituição Federativa de 1988 e as Leis Orgânicas da Saúde de 1990. A descentralização dos serviços de saúde tem sido o eixo norteador para a operacionalização dos princípios organizativos e diretivos do Sistema Único de Saúde. Este estudo apresenta algumas experiências do setor saúde que têm contribuido decisivamente para a materialização desses principios e provocado mudanças importantes no modelo assistencial vigente, bem como têm obtido resultados significativos na produção social de saúde.

\section{PALAVRAS-CHAVE}

Descentralização.

Política de saúde.

Promoção da saúde.

\author{
ABSTRACT \\ The organization of the \\ health sector in Brazil \\ changed significantly after the \\ ratification of the Federal \\ Constitution in 1988 and the \\ Organic Health Laws in 1990. \\ Decentralization of health \\ services has been the basis \\ for the implementation of the \\ organizational and \\ management principles in the \\ Brazilian Unified Health \\ System. This study presents \\ some of the experiences in the \\ health sector that have \\ contributed decisively to the \\ development of such \\ principles, caused important \\ changes in the present care \\ model, and achieved \\ significant results \\ in social health production.
}

\author{
KEYWORDS \\ Decentralization. \\ Health policy. \\ Health promotion.
}

\begin{tabular}{|c|c|}
\hline RESUMEN & ${ }^{*}$ Text \\
\hline La organización del sector & apresentado na prova \\
\hline salud en Brasil presentó & $\begin{array}{l}\text { escrita do concurso } \\
\text { público, no claro de }\end{array}$ \\
\hline cambios significativos después & Professor Doutor, no \\
\hline de la Constitución Federativa & Departamento de \\
\hline de 1988 y las Leyes & $\begin{array}{l}\text { Enfermagem Materno - } \\
\text { Infantil e Saúde }\end{array}$ \\
\hline Orgánicas de Salud de 1990. & Pública da Escola de \\
\hline La descentralización de los & Enfermagem de \\
\hline servicios de salud ha sido el & $\begin{array}{l}\text { Ribeirão Preto da } \\
\text { Universidade de São }\end{array}$ \\
\hline eje norteador en la & $\begin{array}{l}\text { Paulo (EERP/USP) em } \\
\text { janeiro de } 2001 \text {. }\end{array}$ \\
\hline $\begin{array}{l}\text { operacionalizacion de los } \\
\text { princinios oroanizativos } v\end{array}$ & 1 Professor Doutor do \\
\hline $\begin{array}{l}\text { principios organizativos y } \\
\text { directivos del Sistema Único }\end{array}$ & $\begin{array}{l}\text { Departamento de } \\
\text { Enfermagem Materno- }\end{array}$ \\
\hline del Salud. El estudio presenta & Infantil e Saúde \\
\hline algunas experiencias del & $\begin{array}{l}\text { Pública da Escola de } \\
\text { EERP/USP, Centro }\end{array}$ \\
\hline sector salud que han & Colaborador da \\
\hline contribuido en la & Organização Mundial da \\
\hline materialización de estos & desenvolvimento da \\
\hline principios y provocado & pesquisa em \\
\hline cambios importantes en el & $\begin{array}{l}\text { enfermagem. } \\
\text { palha@eerp.usp.br }\end{array}$ \\
\hline $\begin{array}{l}\text { modelo asistencial vigente asi } \\
\text { como en la obtención de }\end{array}$ & $\begin{array}{l}2 \text { Professora Associada } \\
\text { do Departamento de }\end{array}$ \\
\hline resultados significativos en la & $\begin{array}{l}\text { Enfermagem Materno- } \\
\text { Infantil e Saúde }\end{array}$ \\
\hline producción social de salud. & $\begin{array}{l}\text { Pública da EERP/USP, } \\
\text { Centro Colaborador da }\end{array}$ \\
\hline PAL & $\begin{array}{l}\text { OMS para o desenvol- } \\
\text { vimento da pesquisa }\end{array}$ \\
\hline Descentral & em enfermagem. \\
\hline
\end{tabular}

Política de salud.

Promoción de la salud.

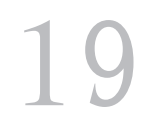

Rev Esc Enferm USP 2003; 37(3):19-26. 
Pedro Fredemir Palha

Tereza Cristina Scatena Villa

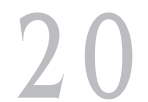

Rev Esc Enferm USP 2003; 37(3): 19-26

\section{APRESENTAÇÃO}

A proposta deste ensaio é abordar a organização da rede básica de saúde no Brasil, a partir da incorporação dos preceitos constitucionais de 1988, que determinou a saúde como um direito de todos e um dever do Estado, mediante políticas públicas e acesso universal. Procuramos apresentar, no processo de reorganização do setor saúde, como a descentralização tem contribuído na qualidade das ações de saúde ofertadas à população brasileira, provocando mudanças importantes nas relações entre os profissionais de saúde e os novos atores sociais oriundos da participação social, corroborando na implantação de novos modelos assistenciais.

A descentralização no campo da saúde pode ser compreendida a partir da relação intrínseca com a gestão do sistema público, especialmente no que se refere à necessidade de aperfeiçoamento das práticas de planejamento, programação, e organização das ações de saúde nos serviços, na busca de práticas sanitárias condizentes com o perfil sanitário e epidemiológico, que impulsionam à construção de alternativas de organização e operacio-nalização de práticas de saúde comprometidas com a melhoria do perfil dos distintos grupos populacionais presentes no Estado brasileiro ${ }^{(1)}$.

Em meados da década de 80 que vivenciamos dois marcos histórico-sociais importantes para a área da saúde, a VIII Conferência Nacional de Saúde e a Constituição Federal de 1988, referenciadas como um grande divisor de águas no que tange atenção à saúde no Brasil ${ }^{(2)}$. Tal legislação incorpora, nos seus conceitos constitucionais, os anseios e as transformações pretendidas no setor saúde, uma busca incessante da base da sociedade em toda a história do movimento da reforma sanitária brasileira ao longo de mais de 30 anos. Nesse sentido, a reforma sanitária não deve ser compreendida apenas como uma reforma setorial, mas sim como um processo social e político em que existe a participação (apoio, adesão) ou não (rejeição, omissão) de diversos seguimentos que compõem a sociedade ${ }^{(3)}$. Portanto, ela incorpora um conjunto de medidas técnicas, administrativas, políticas, culturais e econômicas que podem ser adotadas num determinado contexto histórico, enquanto outras necessitam ser projetadas e implementadas posteriormente.
O Sistema Único de Saúde (SUS) incorporado à Constituição Federativa do Brasil de 1988, através do art. 196, é considerado como uma das maiores conquistas sociais da sociedade brasileira e vem sendo regulamentado a partir de leis orgânicas aprovadas no nível infraconstitucional, procurando regular e materializar os princípios diretivos (universalidade, eqüidade, integralidade) e organizativos (descentralização, regionalização, hierarquização e participação social) do SUS ${ }^{(4)}$. Assim, ao tomarmos como eixo de discussão a descentralização, entendemos que ela rompe com toda a história ditatorial militar que perdurou na nossa sociedade por mais de vinte e cinco anos, permitindo que grande parte do processo decisório seja assumido pelos Estados e municípios ${ }^{(4)}$.

\section{A DESCENTRALIZAÇÃO NO SETOR SAÚDE}

A descentralização tem sua origem nos países desenvolvidos no início dos anos 70, como uma resposta à crise do Estado de BemEstar Social instaurado no pós-guerra, em que a organização e a produção de bens e serviços públicos ou privados eram regulados pelo próprio Estado. Esse Estado de Bem-Estar Social configurou uma nova relação de mediação entre Estado e sociedade, porque, além de assumir a regulação das atividades econômicas, garantiu alguns direitos sociais à população. Assim, a descentralização nesse contexto passa a fazer parte de um conjunto mais amplo de reformas econômicas com intuito racionalizador num Estado que é amplo, centralizador e com presença forte em todas as atividades da vida social e econômica ${ }^{(5)}$.

A descentralização tem suas fortalezas, mas também apresenta suas debilidades ${ }^{(6)}$. No que concerne às fortalezas é possível identificar que elas estão ligadas a uma eficiência maior na alocação dos recursos, em conseqüência da influência dos usuários; ela permite a inovação e adaptação do planejamento e programação às condições regionais $\mathrm{e}$ locais; apresenta maior qualidade e transparência tanto no âmbito alocativo como político; atribui responsabilidade e legitimidade ao gestor na medida em que existe a participação da população no processo de tomada de decisão; e, por fim, pode qualificar melhor a integração entre agências governamentais e não-governamentais reafirmando outro ponto essencial que é a intersetorialidade. Sobre 
as debilidades estão relacionados: a possibilidade de enfraquecimento das estruturas locais, as dificuldades de coordenação e incremento dos custos de transação, a fragmentação dos serviços, a escassez de recursos gerenciais, o clientelismo local e a desestruturação de programas verticais de saúde pública ${ }^{(6)}$.

O processo de descentralização apresenta conflitos e dualidades no que se refere à distribuição de poder e responsabilidades entre os atores federados. Para assumir esse compromisso frente à população torna-se necessário um amadurecimento político em torno de pactos entre atores federados, uma vez que a saúde passa a ser concebida como direito e dever do Estado em seus três níveis de governo, ampliando a responsabilidade da sociedade e gerando resistência pelas dificuldades do nível central em estar delegando poder aos níveis locais. Se por um lado a delegação de poder gera conflitos, por outro existe também muita insegurança em relação aos municípios assumirem parcial ou plenamente novas responsabilidades e atribuições concernentes à gestão e à produção de ações de saúde (promoção, proteção, recuperação e reabilitação) no âmbito local ${ }^{(7)}$. Essa dualidade encontrada nos municípios é fruto da característica do processo de descentralização assumido no Estado brasileiro, através da devolução municipalizada ou seja, com forte centralização do financiamento na União e sem a intermediação e participação dos Estados ${ }^{(6)}$.

No setor saúde brasileiro, o processo de descentralização começa a ser concretizado com a edição das leis orgânicas da saúde que regulamentou o processo de organização dos serviços e a participação social no setor saúde respectivamente ${ }^{(4)}$. Apesar de inúmeros obstáculos que houve ao projeto da reforma sanitária na década de 90 (políticos, econômicos, financeiros, clientelísticos, desrespeito ao arcabouço jurídico-legal, entre outros), a mesma pôde contabilizar importantes avanços no que se refere à organização, gestão, financiamento e ao modelo de atenção no setor saúde ${ }^{(3)}$. Quanto à organização do setor saúde, Paim ressalta as conquistas em relação à descentralização das ações e serviços de saúde com as experiências dos distritos sanitários e consórcios intermunicipais de saúde e a criação da Fundação Nacional de Saúde. Na gestão, aponta aspectos refe- rentes à legislação sanitária (lei 8.080 e 8.142, NOBs 93 e 96) que concretizaram e impulsionaram o processo de municipalização da saúde, a regulação dos planos de saúde, e o funcionamento das conferências e conselhos de saúde.

Em relação ao financiamento, considerado como um dos aspectos mais problemáticos do setor saúde, houve a viabilização do repasse fundo a fundo (do Fundo Nacional de Saúde para os Fundos Estaduais e Municipais de Saúde), o repasse da Contribuição Provisória de Movimentação Financeira (CPMF) e a implantação do Piso Assistencial Básico (PAB - NOB 96). No tocante ao modelo de atenção, o autor destaca os conteúdos das Leis Orgânicas da Saúde, Constituição Federal, Estaduais e Leis Orgânicas Municipais que reforçam as diretrizes e princípios do SUS e a experimentação de modelos assistenciais alternativos tomando como exemplo a vigilância em saúde, as ações programáticas, o programa de saúde da família dentre outros ${ }^{(3)}$.

No que diz respeito à reforma do aparelho do Estado ${ }^{(8)}$, a devolução da União e dos Estados para os municípios e a extinção do INAMPS são pontos relevantes para a consolidação dos princípios doutrinários e organizativos do sistema de saúde, possibilitando a transferência de todo o aparelho técnico-operacional e a infra-estrutura necessária para a operacionalização do sistema de saúde. Dessa forma como estimulou o fomento e desenvolvimento de novos atores sociais na área da saúde, sujeitos possuidores de poder político e de capacidade técnica para exercer um maior controle sobre o sistema público de saúde, através das instâncias colegiadas das Comissões Locais de Saúde e dos Conselhos de Saúde que têm direcionado o exercício do controle social sobre as políticas e ações de saúde no âmbito local ${ }^{(8)}$.

As dificuldades que são identificadas no interior dos Conselhos de Saúde são resquícios ainda das velhas distorções partidárias, clientelísticas ou corporativas de grupos organizados, mas fazendo-se uma avaliação, o resultado global tem sido um movimento democratizador e de relevância no interior dos serviços de saúde, sem precedentes em nenhum outro espaço social da vida nacional ${ }^{(9)}$. No entanto, embora haja essas distorções, o saldo é positivo, pois estão sendo criados
A descentralização como eixo norteador na reorganização e operacionalização dos princípios do Sistema Único de Saúde 
Pedro Fredemir Palha

Tereza Cristina Scatena Villa espaços de ouvidorias independentes nos interiores de várias secretarias estaduais e municipais de saúde como mecanismo do Ministério Público salvaguardar os preceitos constitucionais no tocante às ações e serviços de saúde ${ }^{(7)}$.

Considerando as características do Estado brasileiro, em relação à sua extensa base territorial e profunda desigualdade social que o circunscreve, o processo de descentralização torna-se um aliado importante na minimização da iniqüidade e na produção e oferta das ações de saúde das realidades regionais e locais, na medida em que o planejamento, programação e controle social estejam articulados às realidades tanto dos Estados como dos municípios, possibilitando corrigir as distorções que ocorrem entre municípios e mesmo nas microrregiões como bairros, favelas, vilas urbanas e rurais e tantas outras formas de agrupamentos sociais ${ }^{(10)}$.

Nesse sentido, tem se discutido sobre os avanços e os retrocessos no processo de descentralização nos vários níveis, propulsores ou não de realidades mais condizentes com o local onde são ofertados as ações e os serviços de saúde. Assim, o movimento de descentralização, via municípios, com impulso para o planejamento e organização da produção de ações de caráter coletivo e individual, condizente com as realidades locais, tem contemplado o acúmulo de importantes experiências nos vários cenários, oportunizando a construção de novos modelos assistenciais orientados pelos princípios norteadores do SUS, envolvendo práticas de saúde instrumentalizadas tanto pela saúde coletiva como pela clínica, tendo como produto final a produção social de saúde(11).

Podemos, portanto, considerar que a descentralização, no contexto do setor saúde, é uma ferramenta importante para o desencadeamento de várias intervenções no âmbito da saúde e tem tornado concreto o acesso às ações de saúde por inúmeros sujeitos, antes excluídos da assistência à saúde. Assim, a discussão que empreendemos até este momento nos permite comentar como algumas práticas assistenciais têm contribuído na implementação dos princípios do SUS a partir do processo de descentralização.

\section{AS EXPERIÊNCIAS DOS MODELOS ASSISTENCIAIS NA PRODUÇÃO DE AÇÕES DE SAÚDE}

\section{Modelos Assistenciais Vigentes}

O processo de descentralização tem propiciado importantes experiências inovadoras no sistema de saúde no Brasil denominadas de modelos assistenciais ou modelos de atenção. Entende-se por modelos, as combinações tecnológicas que são estruturadas em decorrência de problemas de saúde (danos e riscos) que definem o perfil epidemiológico de uma população e as necessidades sociais de saúde historicamente definidas ${ }^{(12)}$. Assim, em relação aos modelos de atenção vigentes no setor saúde encontramos o Modelo Médico-Assistencial Privatista e o Modelo Assistencial Sanitarista, dos quais discutiremos as principais características no modo de produção social em saúde, tendo elementos para destacar as diferenças que se apresentam em relação às novas propostas assistenciais experimentadas no setor saúde, nos últimos anos.

O modelo Médico-Assistencial Privatista se apresenta como o mais prestigiado e reconhecido, muito embora não tenha tido capacidade para resolver ou explicar os inúmeros problemas de saúde da população $0^{(3)}$. Esse modelo está presente não somente no setor privado de atenção à saúde, mas também nos filantrópicos e públicos em seus vários níveis de atenção.

Os comentários ${ }^{(3)}$ em relação a esse modelo de assistência dizem respeito a sua forma de organizar sua assistência a partir da demanda espontânea dos usuários dos serviços de saúde, ou seja, reforçam a idéia de que somente mediante o sofrimento ou presença de doença o sujeito deve buscar a atenção, em ordem inversa, quem não sente a necessidade fica fora da cobertura das ações preventivas. O modelo, portanto, somente organiza as ações de saúde para uma dada oferta de atendimento, não se preocupando com a adstrição da clientela, sem o compromisso em obter impactos sobre o nível de saúde da população. Acrescido a isso, não existe preocupação com a organização e acesso às ações e serviços de saúde, levando as instituições públicas a adotar o modelo sanitarista como forma complementar para atender as demandas de cunho coletivo, ou determinados agravos através de campanhas e 
acompanhamento de moléstias infecto-contagiosas como a tuberculose entre outras ${ }^{(3)}$.

No modelo Sanitarista, as ações têm geralmente um caráter temporário e são caracterizadas pela centralidade no planejamento das ações além de exigirem altos investimentos financeiros ${ }^{(3)}$. Têm como característica principal a ação militarista na organização e intervenção proposta e, geralmente, provocam transtornos nos serviços locais, na medida em que se exige uma mobilização importante dos serviços e dos recursos humanos na implementação de ações de caráter coletivo. No que se refere aos programas especiais de saúde pública (saúde da mulher, da criança, tuberculose, hanseníase entre outros) estes normalmente possuem uma administração única e central que não consegue integrar-se ao cotidiano dos serviços de saúde, tendo, portanto, um caráter individual e fragmentado no contexto em que são implantados. Diferem das campanhas pelo caráter mais permanente, porém, ao serem geridos de forma vertical, criam conflitos sérios na ponta do sistema de saúde decorrentes da falta de integração com outras ações e serviços contidos no setor em que os programas estão $\operatorname{alocados}^{(3)}$.

\section{Modelos Assistenciais Alternativos}

Com relação aos modelos alternativos, destacamos aqui para discussão a Oferta Organizada, a Vigilância em Saúde, o Modelo de Defesa da Vida e o Programa de Saúde da Família, com o intuito de discutirmos como eles têm contribuído no processo de descentralização e operacionalização dos princípios constitucionais, que possibilitaram o estabelecimento de novas relações entre usuários e serviços de saúde, organizando a produção, distribuição e equalização das ações de saúde, baseadas nos princípios éticos de valorização da vida e autonomia dos vários sujeitos sociais: trabalhadores de saúde e usuários dos serviços.

A oferta organizada ${ }^{(13)}$, como modelo de estruturação de práticas de saúde, busca superar as velhas formas do processo de trabalho realizadas através dos programas verticais de saúde como o Programa da Tuberculose, da Hanseníase e de gestantes dentre outros. Dessa forma, se constitui um novo modo de compor o trabalho coletivo no serviço de saúde, tendo como finalidade organi- zar o trabalho em saúde, fundamentado no ideal de integração sanitária, ou seja, a articulação de ações médicas com ações coletivas, inspirando-se em tecnologias de base epidemiológica.

Os princípios que nortearam o trabalho da ação programática estão centrados em programas e subprogramas para grupos populacionais e situações específicas com objetivos e finalidades assentados em categorias coletivas, hierarquização interna das atividades profissionais, utilização de equipe multiprofissional, padronização de condutas terapêuticas, utilização de um sistema de informação útil à própria unidade de saúde, adstrição territorial propiciando um impacto e demanda espontânea, bem como regionalização e hierarquização das unidades de saúde. A oferta organizada propõe intervir no plano individual, coletivo em diferentes momentos e amplitudes do processo saúdedoença. Portanto, ao se pensar nas propostas da ação programática é necessário concebê-la como modelo de organização do trabalho em saúde em seu melhor sentido, ou seja, uma proposição de corte técniconormativo, apoiada na busca de um horizonte ético claro e intersubjetivamente aberto, voltada para a construção de práticas de saúde orientadas pelos valores da emancipação humana. Portanto, um modelo assistencial que buscava articular os principais eixos norteadores que viriam conformar o atual sistema de saúde ${ }^{(13)}$.

A vigilância em saúde ${ }^{(2)}$ é compreendida como uma resposta social organizada que responde aos problemas de saúde, a partir da positividade do conceito e da produção social da saúde. Tem como desafio compor as ações de saúde através de um saber interdisciplinar e uma articulação intersetorial possibilitando a organização dos processos de trabalho em saúde, sob a forma de operações, para o enfrentamento, de forma contínua, dos inúmeros problemas presentes num determinado território, tanto no plano coletivo como no individual. Assim, com o intuito de assegurar maior eqüidade e impacto com produção social em saúde, o modelo preconiza a adstrição da população à base territorial. O desenvolvimento das ações de saúde é através da Unidade de Saúde articulando três dimensões importantes: a promoção da saúde, prevenção das enfermidades e acidentes e a atenção curativa ${ }^{(2)}$.
A descentralização como eixo norteador na reorganização e operacionalização dos princípios do Sistema Único de Saúde 
Pedro Fredemir Palha

Tereza Cristina Scatena Villa
O modelo da vigilância em saúde contempla o processo saúde-doença na coletividade, fundamentado na epidemiologia e nas ciências sociais, ao contrário do preconizado pela história natural das doenças que toma o indivíduo e a fisiopatologia como os mais importantes na explicação do processo saúde-doença ${ }^{(14)}$. Assim, a vigilância em saúde ao considerar danos, indícios de danos e de exposição propriamente dita, as várias necessidades e determinantes socioambientais, permite orientar efetivamente as intervenções, abrindo espaços para uma reatualização da reflexão e da ação no que se refere à promoção da saúde e à qualidade de vida ${ }^{(14)}$.

Outra proposta de modelo assistencial é a Defesa da Vida ${ }^{(15)}$ que tem sido operacionalizada no Laboratório de Planejamento do Departamento de Medicina Preventiva da Faculdade de Ciências Médicas da UNICAMP/LAPA, desde a década de 80 . Considera que o modelo não pode se ater somente à rede básica de saúde e deve buscar inovações com outros setores mais complexos da rede de assistência. Propõe, entre outras ações, uma mudança no modelo de gestão dos serviços de saúde mais participativa com o poder distribuído de forma mais horizontalizada. Um dos pontos relevantes dessa proposta tem relação com as principais diretrizes do sistema de saúde proposto no arcabouço jurídico-legal que é a descentralização da gestão dos serviços de saúde, através de colegiados, organizandose atenção à saúde por meio das funções de acolhimento, assistência à saúde e vigilância em saúde. Para o autor, o acolhimento é percebido como um vínculo importante no processo de trabalho, tendo potencial agregador para a responsabilização dos profissionais de saúde na perspectiva de sua autonomia ${ }^{(15)}$.

As duas dimensões do processo de acolhimento são atitudinal e organizativa ${ }^{(16)}$. A primeira corresponde a uma atenção humanizada a todas as pessoas, uma capacidade de escuta acurada dos problemas de saúde uma relação capaz de produzir vínculos entre usuários e equipe de saúde, e uma resposta sempre positiva das demandas trazidas pela população. A segunda mantém uma aproximação com o território de adscrição, estabelecendo os fluxos e contrafluxos dos usuários para com a unidade de saúde, evitando respostas negativas às necessidades demandadas e à implantação da avaliação em serviço ${ }^{(16)}$.
O Programa de Saúde da Família $(\mathrm{PSF})^{(4)}$, proposto pelo Ministério da Saúde, tem como fundamentação filosófica e teórica os princípios do SUS. A estratégia de Saúde da Família, mais comumente usada, tem como base, no processo de trabalho, a reorientação das ações de saúde por problemas, procurando contemplar os princípios da integralidade, intersetorialidade, planejamento e programação local, hierarquização, co-responsabilidade e adstrição da população em um determinado território. Para a operacionalização do processo de trabalho, o programa propõe atuação em equipe como mecanismo de propiciar a integralidade da assistência e diminuição do processo de fragmentação do trabalho. Nesse sentido, segundo o Ministério da Saúde, o PSF tem como objetivo contribuir para a reorientação do modelo assistencial, a partir da atenção básica, com definição de responsabilidades entre os serviços de saúde e os usuários, no sentido de equacionar de maneira mais racional e ética as necessidades da população de seu território ${ }^{(4)}$.

Apesar da relevância da ampliação do PSF, proposto pelo Ministério da Saúde, ainda faltam evidências que apontem tal programa como uma estratégia suficiente e capaz de reorientar o modelo de assistência ainda vigente. Não se negam as experiências positivas encontradas em vários municípios brasileiros, mas acredita-se que essa estratégia ainda está muito distante de alterar o "SUS real" e de ter capacidade para transformar o atual sistema de saúde no Brasil ${ }^{(3)}$.

\section{À GUISA DAS DISCUSSÕES}

As produções de ações de saúde, pelos diferentes modelos assistenciais que se fazem presentes no território brasileiro apresentam diferenças e resultados impactantes sobre o processo de saúde-doença e sobre a produção social de saúde. Se olharmos os modelos assistenciais ainda vigentes e com forte conotação sobre o retorno ou devolução social de sua produção, podemos constatar que tanto o modelo médico-assistencial privatista quanto o sanitarista não concentram suas atenções no controle de certos agravos ou sobre alguns grupos mais susceptíveis ao adoecimento ou a morte ${ }^{(3)}$. São modelos que não se preocupam com os determinantes mais gerais do quadro sanitário ou mesmo com os problemas do sistema de serviços de saúde. Em relação ao nosso 
objeto de análise, a descentralização e os princípios do SUS, os modelos vigentes não tem se preocupado com a questão do processo de descentralização, com a integralidade da assistência e não procura envolver o processo de participação popular ou mesmo o estímulo ao controle social ${ }^{(3) .}$

Os mecanismos jurídicos ${ }^{(17)}$, NOBs e legislação infraconstitucional acabaram exercendo papéis fundamentais para adoção de estratégias que possibilitaram a regulação e indução do processo de descentralização, na definição de repasse de recursos financeiros entre a União, Estados e Municípios e na pactuação entre os vários atores sociais. A descentralização assumiu, portanto, diferentes formatos, no Brasil, que são oriundos das potencialidades presentes na estrutura organizacional, da articulação entre os serviços, das condições políticas, financeiras, gerenciais e administrativas presentes nos diversos cenários municipais, influenciando importantes mudanças na prática médica e no ênfase às atividades ambulatoriais, possibilitando aumentar a produção social em saúde através de novas práticas assistenciais ${ }^{(17)}$.
A descentralização do SUS que ocorreu através do processo de municipalização imputou novos desafios e responsabilidades para Estados e municípios em relação ao desenvolvimento da atenção primária, provocando quadro de expansão da cobertura através da criação de novas unidades básicas de saúde no âmbito local ${ }^{(16)}$. É salutar apontar que no Brasil existem essas experiências e reflexões construídas a partir dos processos de distritalização, possibilitando que a descentralização do setor saúde tenha outros rumos que não a simples descon-centração de recursos ou apenas como prestador de serviços por parte dos atores e gestores municipais $^{(12)}$.

Muitos entre os distintos projetos apresentam similaridades, convergências, divergências ou mesmo complementaridades. No entanto, o que importa para o campo da saúde coletiva é a riqueza das propostas alternativas que podem assumir um caráter de enfrentamento do modelo hegemônico e sua completa reforma, tanto no plano teórico quanto no político.

\section{REFERÊNCIAS}

(1) Teixeira C. O futuro da prevenção. Salvador, Casa da Qualidade Editora; 2001.

(2) Mendes EV. Uma agenda para a saúde. São Paulo: Hucitec; 1996.

(3) Paim JS. A reforma sanitária e os modelos assistenciais. In: Rouquayrol MZ, Almeida Filho N. Epidemiologia e saúde. $5^{\text {a }}$ ed. Rio de Janeiro: MEDSI, 1999. p. 473-87.

(4) Ministério da Saúde. Secretaria de Assistência à Saúde da Família. SUS - Conquistas. Brasília; 2000.

(5) Ministério da Saúde. Conselho Nacional dos Secretários Municipais de Saúde. Lei No 8.080 de 19 de Setembro de 1990. Dispõe sobre as condições para a promoção, proteção e recuperação da saúde, a organização e o funcionamento dos serviços correspondentes, e dá outras providências. Legislação Básica do SUS. Brasília; 1990.
(6) Ministério da Saúde. Conselho Nacional dos Secretários Municipais de Saúde. Lei No 8.142 de 28 de Dezembro de 1990. Dispõe sobre a participação da comunidade na gestão do Sistema Único de Saúde - SUS e sobre as transferências intergovernamentais de recursos financeiros na área da saúde e dá outras providências. Legislação Básica do SUS. Brasília; 1990.

(7) Mendes EV. Os grandes dilemas do SUS: Tomo II. Salvador: Casa da Qualidade Editora; 2001.

(8) Mendes EV. A descentralização do sistema dos serviços de saúde no Brasil, novos rumos e um novo olhar sobre o nível local. In: Mendes EV; organizador. A organização da saúde no nível local. São Paulo: Hucitec; 1998.

(9) Carvalho GCM. O movimento atual do SUS. A ousadia de cumprir e fazer cumprir a lei. Saúde Sociedade 1993; 2 (1) :9-24.

(10) Conselho Nacional de Saúde. Desenvolvimento do sistema único de saúde no Brasil: avanços, desafios e reafirmação dos princípios e diretrizes. Saúde Debate 2002; 26(62): 295-310.
A descentralização como eixo norteador na reorganização e operacionalização dos princípios do Sistema Único de Saúde 
Pedro Fredemir Palha

Tereza Cristina Scatena Villa
(11) Costa WGA, Maeda ST. Repensando a rede básica do SUS e o distrito sanitário. Saúde Debate 2001; 25(57):15-29.

(12) Paim JS. Políticas de descentralização e atenção primária à saúde. In: Rouquayrol MZ, Almeida Filho N. Epidemiologia e saúde. $5^{\text {a }}$ ed. Rio de Janeiro: MEDSI; 1999. p. 489503.

(13) Nemes MIB. Prática programática em saúde. In: Schraiber LB, Nemes MIB, Mendes-Gonçalves RB. Saúde do adulto, programas e ações na unidade básica. São Paulo: Hucitec; 2000. p. $48-65$.
(14) Paim JS, Almeida Filho N. A crise da saúde pública e a utopia da saúde coletiva. Salvador: Casa da Qualidade Editora; 2000.

(15) Campos GWS. Desafios para novos modelos de gestão: prioridade é fortalecer a rede básica.Temas. 1997. p.15:23.

(16) Mendes EV. A atenção primária à saúde no SUS. Fortaleza: Escola de Saúde Pública do Ceará; 2002.

(17) Siqueira SAV, Senna MCM, Oliveira PTR, Pinto LF. Descentralização e assistência à saúde no Brasil: a oferta de serviços nos anos 90. Saúde Debate 2002; 26 (60):25-36. 